\title{
Eine bibliometrische Analyse des Forschungsstandes und der zukünftigen Forschungsfragen für die Transformation vom Produzenten zum Dienstleister
}
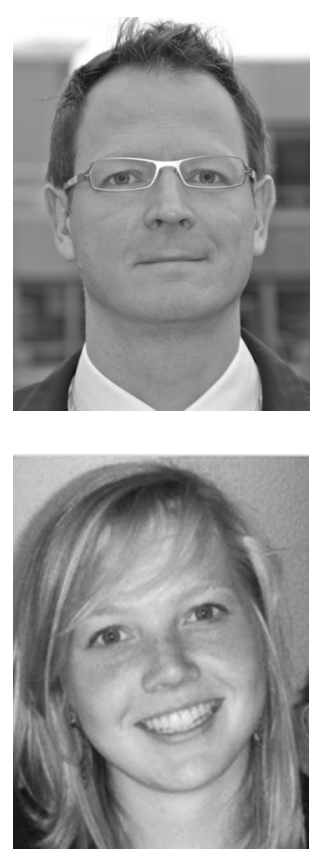

\section{Heiko Gebauer und Caroline Saul}

Produzierender Dienstleister, Lösungsanbieter, Literaturanalyse, intellektueller Kern, zukünftige Forschungsthemen

Service provider, Solution providers, Literature analysis, Intellectual core, Future research direction.

Die Transformation vom Produzenten zum Dienstleister zählt zu den prominenten Forschungsthemen mit einer kontinuierlich steigenden Anzahl an wissenschaftlichen Artikeln. Wir analysieren die Beiträge zum Forschungsthema und identifizieren den intellektuellen Kern. Der intellektuelle Kern besteht aus sieben Forschungsrichtungen: Erbringen von Lösungen, Vermarktung von Lösungen, Dienstleistungen in produzierenden Unternehmen, Erfolg im Dienstleistungsgeschäft, Produkt-Service-Systeme und Dienstleistungsentwicklung. Wir artikulieren einige der kritischen empirischen und theoretischen Bedenken. Forschung kann von der Ausnutzung der Synergien und der Kombination der Forschungsrichtungen profitieren. Wir zeigen konkrete Wege zur Erweiterung und Vertiefung der empirischen und theoretischen Forschung auf.

The transition from products to services is popular research theme with a continuously increasing number of articles. We analyze these articles and identify the intellectual core. The intellectual core consists of seven research directions: Solution delivery, Solution marketing, Services in manufacturing companies, Service performance in manufacturing companies, product-service-systems, and industrial service development. We articulate critical empirical and theoretical considerations. Future research should further explore the synergies among the research directions. We articulate concrete research questions to expand and to deepen the empirical and theoretical research.

\section{Motivation}

Die Transformation vom Produzenten zum produzierenden Dienstleister zählt zu den prominenten Forschungsthemen im Dienstleistungsbereich (Jacob/Ulaga 2008; Ostrom et al. 2010; Kunz/Hogreve 2011). Die kontinuierlich steigende Anzahl an wissenschaftlichen Artikeln veranschaulicht die schnelle Entwicklung des Themas (vgl. Abbildung 1). Das Thema ist ebenfalls von sehr hoher Praxisrelevanz. Produzierenden Unternehmen ergänzen ihre Produkte mit Dienstleistungen und investieren in den Ausbau des Dienstleistungsge- 
schäfts. Der Ausbau des Dienstleistungsgeschäfts betrifft Klein- und Mittlere Unternehmen (KMUs) und Multinationale Unternehmen (MNUs) sowie Unternehmen, die einfache Produkte oder komplexe Systeme anbieten. Unternehmen wie ABB, Caterpillar, General Electric, IBM, Siemens usw. wachsen vorwiegend im Dienstleistungsgeschäft und der Umsatzanteil von Dienstleistung ist heute circa 50 Prozent.

Das Forschungsthema basiert auf der Annahme, dass sich Unternehmen vom Produzenten zum produzierenden Dienstleister weiterentwickeln. Produzenten sind Produktverkäufer, bei denen Dienstleistungen ein Add-on zum Produkt darstellen. Produkte sind verantwortlich für den Unternehmenserfolg und garantieren Wachstum, Umsatz, Gewinn, Wettbewerbsdifferenzierung und Kundenzufriedenheit. Bei einem produzierenden Dienstleister hängt der Unternehmenserfolg von den Dienstleistungen ab. Dienstleistungen sind ein essentieller Teil vom Umsatz und Gewinn, wesentlicher Eckpfeiler für Wachstum und Wettbewerbsdifferenzierung und sichern die Kundenzufriedenheit. Dienstleistungen werden mit den Produkten kombiniert und in kundenspezifische Lösungen integriert (Davies 2004; Sawhney et al. 2004; Tuli et al. 2007; Ulaga/Reinartz 2012).

Die wissenschaftlichen Beiträge und die Praxisrelevanz lassen vermuten, dass gemeinsame theoretische Grundlagen und umfangreiche empirische Untersuchungen existieren. Bisher fehlen jedoch allgemeine theoretische Grundlagen und vollständig empirisch überprüfte Zusammenhänge. In der Englischen Literatur wird der Wandel vom Produzenten zum produzierenden Dienstleister als Transition from Products to Services, Service Infusion, Product-Service Systems, Solution Provider oder Servitization konzeptionalisiert (Vandermerwe/Rada 1988; Oliva/Kallenberg 2003; Davies 2004; Tukker 2004; Neu/Brown 2005; Windabl/Lakemond 2006; Gebauer et al. 2010; Ulaga/Reinartz 2012). In der deutschsprachigen Literatur finden sich Begriffe wie hybride Leistungsbündel, industrielle Dienstleistungen oder Systemanbieter (Belz et al. 1997; Backhaus 2003). Die Variationen in der Konzeptionalisierung tragen zur Reichhaltigkeit der Forschung bei. Sie schränken aber auch notwendige theoretische Fortschritte und empirische Untersuchungen ein. Diese Einschränkungen können sich auflösen, wenn sich das Forschungsthema weiterentwickelt. Dennoch besteht das Risiko, dass wir uns in einer verzerrten Forschungslandschaft bewegen und unklaren Forschungsrichtungen folgen. Eine Strukturierung der Forschungsbeiträge sowie eine Untersuchung der Entwicklung der Forschungslandschaft kann zur Weiterentwicklung beitragen.

In dem vorliegenden Beitrag analysieren wir die bestehende Literatur mit Hilfe einer bibliometrischen Methode und formulieren Handlungsempfehlungen für die Weiterentwicklung des Forschungsthemas. Wir beginnen den Beitrag mit einer Beschreibung der bibliometrischen Methode. Anschliessend präsentieren wir die Resultate unserer bibliometrischen Analyse. Aufbauend auf dieser Analyse formulieren wir Handlungsempfehlungen für die zukünftige Ausgestaltung des Forschungsthemas. Abschliessend fassen wir die wichtigsten Erkenntnisse des Artikels kurz zusammen.

\section{Bibliometrische Methode}

Bibliometrische Methoden werden zur Entdeckung und Artikulation der Strukturen innerhalb von Forschungsgebieten genutzt (Nerur et al. 2008). Wir wählten zuerst eine geeignete Literaturdatenbank aus. Web of Science beinhaltet weniger wissenschaftliche Zeitschriften als Scopus. Die Verwendung von Google Scholar wurde verworfen, da es keine ausreichende Suchfunktion hat und im Gegensatz zu Scopus keine Identifikationscodes der Arti- 
kel beinhaltet. Scopus ist aus diesen Gründen am besten geeignet. Wir grenzten den Zeitraum auf 1987 bis 2012 ein.

Anschliessend identifizierten wir die Schlüsselwörter für das Thema Dienstleistungen in produzierenden Unternehmen. Die Schlüsselwörter umfassen: service infusion, productservice-systems, transition from products to services, solution providers, service business development, servitization, after-sales service und system suppliers. Nach diesen Schlüsselwörtern suchten wir in den Feldern: Title, Abstract und Key Words. Aus der resultierenden Liste wurden Artikel mit vielen Zitationen identifiziert. Wir wählten zusätzlich die Beiträge aus, welche diese Artikel zitieren. Hieraus ergab sich eine weitere Liste an relevanten Artikeln. Beide Listen wurden miteinander kombiniert und hinsichtlich von Inkonsistenzen überprüft.

Zwei unabhängige Gutachter evaluierten den Inhalt jedes Artikels. Das resultierende Cohen's Kappa als Indikator der Interrater-Reliabilität beträgt ausreichende 0,81 (Perreault/Leigh 1989). Die Evaluierung führte zu 676 Artikel zum Thema Dienstleistungen in produzierenden Unternehmen. Wir analysierten diese Beiträge hinsichtlich der Anzahl pro Jahr, der wissenschaftlichen Zeitschriften, der Schlüsselworte und Länder aus denen die Beiträge kommen.

Es folgte eine Zitationsanalyse. Die Anzahl der Zitationen ist ein geeigneter Indikator zur Identifizierung des intellektuellen Kerns eines Forschungsthemas (Culnan 1986). Alle Artikel mit mehr als 25 Zitationen bilden den intellektuellen Kern. Anschliessend wurde eine deskriptive Analyse dieses intellektuellen Kerns durchgeführt. Wir kodierten die Artikel anhand folgende Kategorien: (1) wissenschaftlicher Artikel versus Managementbeitrag, (2) konzeptioneller versus empirischer Artikel, (3) quantitative versus qualitative Daten, (4) Methoden zur Datensammlung (z.B. Interviews, Observationen oder Aktionsforschung), (5) Zeithorizont der Datensammlung (kurzfristig versus langfristig), (6) Untersuchungsgegenstand (z.B. produzierende Unternehmen, Zuliefer-Kundenbeziehung, Kunden von produzierende Unternehmen) und (7) Industriebranchen.

Ferner führten wir eine Ko-Zitationsanalyse durch. Für die Analyse nutzen wir den relativen Ko-Zitationswert als Mass für die Ähnlichkeit von zwei Artikeln. Die Ko-Zitationswerte wurden mit Hilfe des Salton's Kosinus-Masses in eine Korrelationsmatrix überführt. Dieses Mass ermöglicht eine Standardisierung von Daten und eine Reduktion der Nullen in der Ko-Zitations- bzw. Korrelationsmatrix (Leydesdorff 2005). Diese Korrelationsmatrix dient als Input für die Strukturierung des Forschungsgebietes durch eine Multidimensionale Skalierung (MDS) und Faktoranalyse.

Wir nehmen an, dass die einzelnen Forschungsrichtungen innerhalb des Themengebietes nicht unabhängig sind und nutzen eine Varimaxrotation und Hauptkomponentenanalyse. Mit dieser Vorgehensweise klassifizieren wir die Artikel in Gruppen, genannt Faktoren, auf Basis der variierenden Ähnlichkeiten zwischen den Artikeln. Jeder Faktor bildet eine relativ homogene Gruppe von Artikeln. Diese Gruppe interpretieren wir als eigene Forschungsrichtung. Die beiden oben genannten unabhängigen Gutachter beurteilten, ob die Artikel eine homogene Forschungsrichtung bilden oder nicht.

Eine MDS ergänzte die Faktorenanalyse. Die MDS ermöglicht eine grafische Konfiguration der inhaltlichen Nähe der Artikel innerhalb des intellektuellen Kerns. Die Position eines Artikels hängt von der inhaltlichen Nähe zu den anderen Artikeln ab. Wir erarbeiteten eine inhaltsbasierte Interpretation der Achsen dieser grafischen Konfiguration. Diese Inter- 
pretation nutzt die Position der Faktoren in der grafischen Konfiguration und die relative Position der einzelnen Artikel entlang der Achsen.

\section{Resultate der bibliometrischen Analyse}

\subsection{Deskriptive Resultate}

Europäische Beiträge prägen das Forschungsthema. Auch wenn mit 15,9 Prozent die meisten Beiträge aus den USA stammen, steht dem ein Anteil von 61,6 Prozent aus Europa gegenüber. Deutschsprachige Forschung spielt eine sehr vitale Rolle (z.B. Deutschland mit 8,9 Prozent und Schweiz mit 5,4 Prozent). Unter den weiteren Europäischen Ländern sind vor allem Regionen mit ausgeprägten produzierenden Industrien vertreten. Beispiele sind die Beiträge aus Frankreich (3,8 Prozent), Italien (4,0 Prozent), Grossbritannien (13,8 Prozent) oder die skandinavischen Länder Schweden (8,4 Prozent) und Finnland (8,0 Prozent). In den letzten Jahren gibt es eine zunehmende Anzahl an Beiträgen aus China (6,2 Prozent), Südkorea (1,8 Prozent), Taiwan (1,9 Prozent) oder Japan (1,3 Prozent).

Die Analyse der Anzahl und der Inhalte der Artikel zeigt zwei Wendepunkte in der Entwicklung des Forschungsthemas (vgl. Abbildung 1). Diese Wendepunkte unterteilen die Entwicklung in drei Phasen. Phase 1 beginnt mit den Beiträgen Ende der 1980er Jahre und geht bis 1999. Dominierende Schlüsselwörter sind Kundendienst, industrielle Dienstleistungen und Kunden-Lieferanten-Beziehungen. Die Artikel sehen Dienstleistungen in produzierenden Unternehmen als eingebettet in das Investitionsgütermarketing. Beispiele sind die frühen Arbeiten zur Entwicklung von industriellen Dienstleistungen (Brentani 1989) oder zur Dienstleistungsorientierung in produzierenden Unternehmen (Bowen et al. 1989; Martin/Horne 1992).

\section{Anzahl der Artikel}

Phase 3: Reifephase des

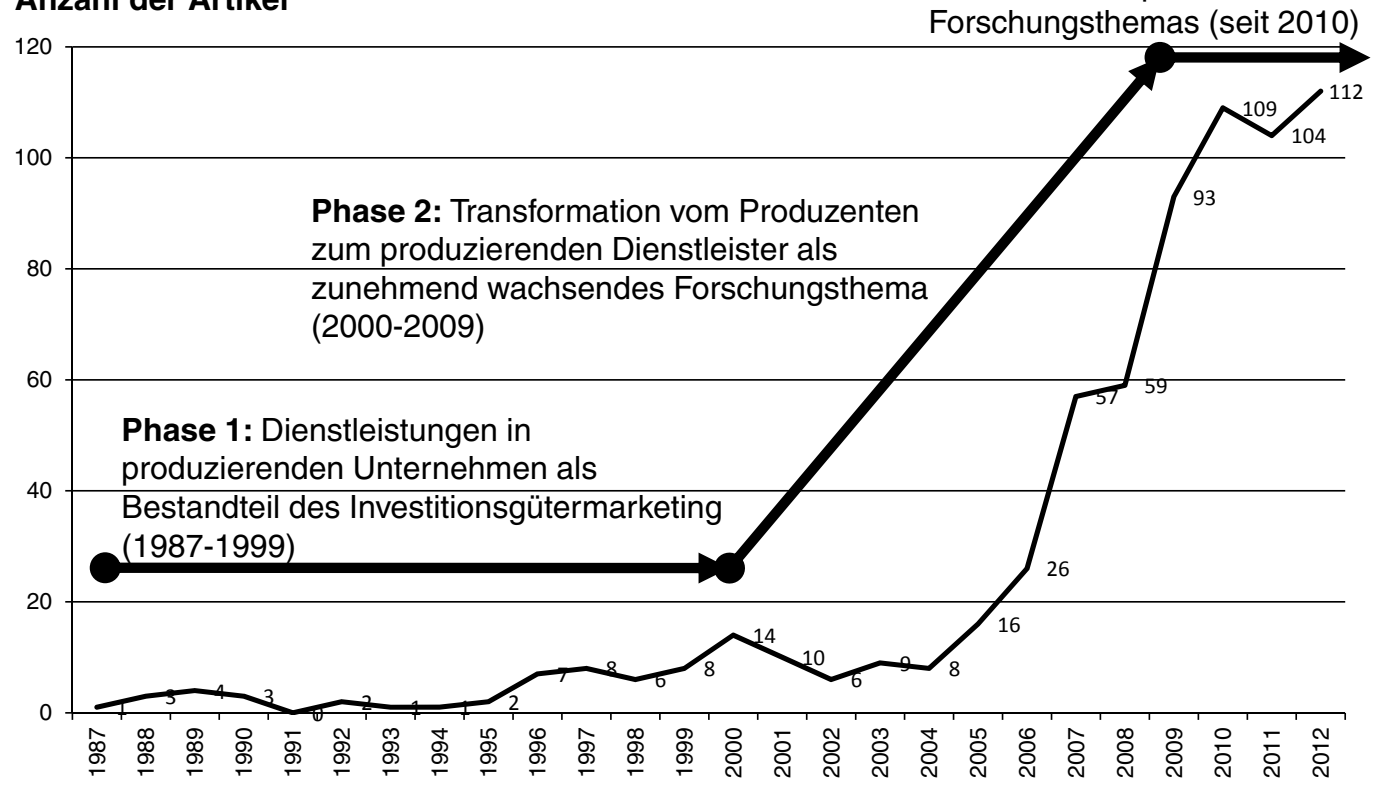

Abbildung 1: Entwicklungsphasen 
Phase 2 beginnt im Jahr 2000 und endet 2009. In Phase 2 entwickelt sich das Thema in ein eigenständiges, rasant wachsendes Forschungsgebiet. Die wichtigen Konzeptionalisierungen wie Product-Service-Systems, Transition line from Products to Services, Service Business Development oder Solution Providers entstehen in dieser Phase (z.B. Mathieu 2001a; Oliva/Kallenberg 2003; Davies 2004; Tukker 2004; Gebauer et al. 2005). Eine Ausnahme bildet der Begriff Servitization, welcher auf Vandermerwe und Rada (1988) zurückgeht. In Phase 2 wird der Begriff Servitization vor allem durch britische Autoren wieder aufgegriffen. Der Fokus aus Phase 1 auf Kundendienst, Kundenzufriedenheit und Investitionsgütermarketing wird ersetzt durch andere Dienstleistungskategorien (z.B. AfterSales-Service, Value-added Services, produkt- und kundenunterstützende Dienstleistungen). Es liegt ebenfalls ein stärkeres Gewicht auf der Dienstleistungsprofitabilität, dem Dienstleistungsumsatz und dem Wachstum durch Dienstleistungen.

Phase 3 begann im Jahr 2010 und kann als erste Reifephase bezeichnet werden. Von 2010 bis 2012 erschienen etwas mehr als 100 Artikel pro Jahr. Die Reifephase kennzeichnet sich durch zwei Eigenschaften. Erstens, der Erkenntnisgewinn in den einzelnen Beiträgen nimmt ab. Die Artikel bestätigen die bisherigen Erkenntnisse und erweitern das theoretische und empirische Wissen nur relativ wenig. Zweitens, nur wenige Beiträge betrachten das Thema aus weiteren theoretischen Perspektiven. Hierzu zählen Perspektiven wie die Konzeptualisierung des Wandels vom Produkt zur Dienstleistung als Geschäftsmodellinnovation (Kindstrom 2000) oder die Einbettung der Dienstleistungsorientierung in Innovationsfähigkeit und Marktorientierung (Gebauer et al. 2011). Die Mehrheit der Artikel führen die theoretischen Konzepte der Phase 2 fort. Eine Vertiefung und Ausweitung der theoretischen Konzepte findet nur selten statt.

\subsection{Beschreibung des intellektuellen Kerns}

55 Publikationen mit mehr als 25 Zitationen interpretieren wir als den intellektuellen Kern (vgl. Tabelle 1). Diese Publikationen umfassen 60,2 Prozent der gesamten Anzahl an Zitationen. Aufgrund der hohen Relevanz für die Praxis haben Managementbeiträge (z.B. Harvard Business Review, Sloan Management Review usw.) mit 15,9 Prozent einen relativ hohen Anteil am intellektuellen Kern. Dies unterscheidet das Forschungsthema von anderen Themen (Nerur et al. 2008). Der intellektuelle Kern der Diskussion um die dynamischen Fähigkeiten im Unternehmen beinhaltet beispielsweise keinen einzigen Managementbeitrag (Stefano et al. 2010). Dynamische Fähigkeiten als Grundlage zur Anpassung an veränderte Bedingungen im Umfeld haben zwar ebenfalls eine hohe praktische Relevanz. Entsprechende Managementbeiträge scheinen jedoch nicht zum intellektuellen Kern beizutragen.

Artikel Anzahl der Zitationen Zitationen pro Jahr (Ende 2012)

(Ende 2012)

Oliva/Kallenberg (2003)

327

36

Vandermerwe/Rada (1988)

170

7

Tuli et al. (2007)

154

31

Brentani (1989)

Davies (2004) 


\begin{tabular}{|c|c|c|}
\hline Artikel & $\begin{array}{l}\text { Anzahl der Zitationen } \\
\text { (Ende 2012) }\end{array}$ & $\begin{array}{l}\text { Zitationen pro Jahr } \\
\text { (Ende 2012) }\end{array}$ \\
\hline Wise/Morrison (1999) & 114 & 10 \\
\hline Tukker (2004) & 110 & 14 \\
\hline Mathieu (2001a) & 109 & 10 \\
\hline Mathieu (2001b) & 103 & 9 \\
\hline Galbraith (2002) & 98 & 10 \\
\hline Brentani (1995) & 91 & 5 \\
\hline Cohen et al. (2006) & 89 & 15 \\
\hline Gebauer et al. (2005) & 88 & 13 \\
\hline Sawhney et al. (2004) & 86 & 11 \\
\hline Neu/Brown (2005) & 85 & 12 \\
\hline Quinn et al. (1990) & 84 & 4 \\
\hline Davies et al. (2006) & 79 & 13 \\
\hline Davies et al. (2007) & 76 & 15 \\
\hline $\operatorname{Brax}(2005)$ & 70 & 10 \\
\hline Brady et al. (2005) & 67 & 10 \\
\hline Tukker/Tischner (2006) & 65 & 11 \\
\hline Cova/Salle (2008) & 60 & 15 \\
\hline Roy (2000) & 59 & 5 \\
\hline Windabl/Lakemond (2006) & 55 & 9 \\
\hline Matthyssens/Vandendempt (2008) & 53 & 13 \\
\hline Neely (2008) & 53 & 18 \\
\hline Jacob/Ulaga (2008) & 52 & 13 \\
\hline Vandendempt/Matthyssens (1998) & 51 & 4 \\
\hline Jackson et al. (1995) & 48 & 3 \\
\hline Baines et al. (2009) & 46 & 15 \\
\hline Gebauer (2008) & 45 & 11 \\
\hline Fang et al. (2009) & 44 & 15 \\
\hline Boyt/Harvey (1997) & 43 & 3 \\
\hline Markeset/Kumar (2003) & 42 & 5 \\
\hline Slack et al. (2004) & 41 & 5 \\
\hline Allmendinger/Lombreglia (2005) & 41 & 6 \\
\hline Pettinen/Palmer (2007) & 40 & 8 \\
\hline Van Birgelen et al. (2002) & 39 & 4 \\
\hline Gebauer/Friedli (2005) & 38 & 5 \\
\hline Jackson/Cooper (1988) & 37 & 2 \\
\hline
\end{tabular}


Artikel

Anzahl der Zitationen

(Ende 2012)
Zitationen pro Jahr

(Ende 2012)
Johnson/Olhage (2004)

Homburg et al. (2003)

Spring/Arouju (2004)

Droege et al. (2009)

Gebauer et al. (2006)

Neu/Brown (2008)

Martin/Horne (1992)

Thomas (2004)

Wise/Baumgartner (1998)

Gebauer et al. (2010)

Antioco et al. (2008)

Malleret (2006)

Voss (2005)

Edvardsson et al. (2008)

\section{7}

35

35

34

33

32

31

31

29

28

28

27

26

25

\section{5}

4

12

11

6

8

2

4

2

14

7

5

4

6

Tabelle 1: Intellektuelle Kern

Die wissenschaftlichen Beiträge (84,1 Prozent) sind im Wesentlichen empirische Beiträge (63,6 Prozent). Konzeptionelle Artikel (18,2 Prozent) und Artikel mit einem Literaturüberblick (2,3 Prozent), welche normalerweise einen wesentlichen Teil eines intellektuellen Kerns bilden, sind in der Minderheit. Die empirischen Beiträge (63,6 Prozent) sind mit 45,4 Prozent mehrheitlich qualitative Studien auf Basis einzelner Interviews. 18,2 Prozent der Artikel basieren auf quantitative Studien.

Der betrachtete Zeitraum ist in der Regel kurzfristig. Neu und Brown (2008) betrachten einen Zeitraum von 18 Monaten, Davies (2004), Davies et al. (2006) sowie Matthyssens und Vandenbempt (2008) einen Zeitraum von fünf bis acht Jahren. Nur wenige der Beiträge messen systematisch den Beitrag von Dienstleistungen zum Unternehmenserfolg. So fehlt eine präzise Darstellung der Veränderungen im Anteil des Dienstleistungsumsatzes am gesamten Umsatz des Unternehmens und die Auswirkung auf den Unternehmenserfolg ist unklar. Es wird argumentiert, dass Dienstleistungen profitabler sind als Produkte. Der Ausbau des Dienstleistungsangebots erhöht den Dienstleistungsumsatz, welcher wiederum den Unternehmenserfolg steigert. Empirische Daten zur Profitabilität von Dienstleistungen werden nur in wenigen Artikeln dargestellt.

Hier ist ebenfalls das Service Paradox zu erwähnen (Brax 2005; Gebauer et al. 2005). Service Paradox bedeutet, dass Unternehmen in den Ausbau des Dienstleistungsgeschäfts investieren, aber die erwarteten Steigerungen im Dienstleistungsumsatz und -gewinn nicht realisieren. Trotz des häufig genannten Arguments, dass Unternehmen Schwierigkeiten bei der Transformation haben und sich dem Service Paradox gegenüber stehen, die meisten Fallstudien sind von erfolgreichen Unternehmen.

Einzelne quantitative Studien verwenden dienstleistungs- und unternehmensbezogene Kennzahlen. Homburg et al. (2003) untersuchen den kausalen Zusammenhang zwischen 
dem finanziellen und nicht-finanziellen Beitrag von Dienstleistungen und dem Unternehmenserfolg. Fang et al. (2008) testen die Beziehung zwischen dem Anteil des Dienstleistungsumsatzes am Gesamtumsatz und dem Unternehmenswert. Antioco et al. (2008) verwenden produkt- und dienstleistungsbezogene Erfolgskennzahlen. Dienstleistungsbezogene Erfolgskennzahlen werden operationalisiert durch den Anteil des Dienstleistungsumsatzes. Produktbezogene Erfolgskennzahlen werden durch den Produktumsatz im Verhältnis zum Wettbewerber gemessen.

Der intellektuelle Kern umfasst eine Vielzahl von Industrien. Investitionsgüterhersteller aus dem Maschinen- und Anlagenbau wurden am häufigsten untersucht. Weitere Industrien sind Unternehmen aus dem Bereich Mikroelektronik, technische Dienstleister, Händler, Hersteller elektrischer und mechanischer Komponenten, die Metallindustrie sowie Anbieter von Informations- und Kommunikationstechnologien (Matthyssens/Vandendempt 1998; Mathieu 2001b; Homburg et al. 2003; Neu/Brown 2005).

Die Mehrzahl der Unternehmen ist als Hersteller von Endsystemen in der Wertschöpfungskette positioniert. In den drei Artikeln, Oliva und Kallenberg (2003), Davies (2004) und Gebauer et al. (2005) ist beispielsweise nur ein Unternehmen kein Hersteller von Endsystemen. Hersteller von Endsystemen haben einen direkten Zugang zum Betreiber der Endsysteme. Sie haben deswegen einen Vorteil beim Ausbau des Dienstleistungsgeschäfts. Zulieferunternehmen, welche dem Hersteller der Endsysteme vorgelagert sind, haben diesen Zugang nicht und stehen deswegen vor anderen Herausforderungen. Die Artikel diskutieren vorrangig den Ausbau des Dienstleistungsgeschäfts in grösseren Unternehmen. Es existieren nur wenige Beiträge, die sich auf die speziellen Herausforderungen von KMUs konzentrieren.

Die Mehrheit der empirischen Beiträge verwendet keinen ausgeprägten theoretisch-fundierten Rahmen zur Datenaufnahme und -analyse. Neu und Brown (2005) verwenden die Kontigenztheorie zur Erklärung der Auswirkung der Anpassungen zwischen dem Umfeld, der Strategie und den organisationalen Faktoren (Prozesse, Kompetenzen, Strukturen und Anreizsysteme) auf den Unternehmenserfolg. Gebauer et al. (2005) benutzen die ValenzInstrumentalitäts-Erwartungs-Theorie als Konzeptionalisierung für die Motivation zum Ausbau des Dienstleistungsgeschäft. Tuli et al. (2007) konzeptionalisiert kundenspezifische Lösungen als Kombinationen aus Produkten und Dienstleistungen und als ein Bündel von Beziehungsprozessen zwischen Kunden und Lieferanten. Das Bündel umfasst die Definition der Kundenanforderungen, kundenspezifische Anpassungen und Integration von Produkten und Dienstleistungen, deren Implementierung, und Lebenszyklusunterstützung. Diese Beziehungsprozesse zielen darauf ab, die operativen und strategischen Bedürfnisse der Kunden zu identifizieren und zufriedenzustellen. Penttinen und Palmer (2007) kombinieren die Art der Kunden-Lieferanten-Beziehung (transaktions- oder beziehungsorientiert) mit dem Dienstleistungsangebot. Trotz dieser Beispiele stellen wir fest, dass die Mehrheit der empirischen Beiträge keinen theoretisch-fundierten Rahmen zur Datenaufnahme und -analyse verwenden.

Grundsätzlich gehen die Beiträge davon aus, dass Dienstleistungen eine Differenzierungsstrategie darstellen. Artikel zur Rolle von Dienstleistungen zur Unternehmensstrategie unterscheiden in verschiedene Dienstleistungsstrategien. Diese Strategien ähneln den produkt-, nutzer- oder ergebnisorientierten Produkt-Service-Systemen (Tukker 2004). Es existiert ein Vielzahl von Dienstleistungsstrategien und Autoren verwenden unterschiedliche Begriffe für sehr ähnliche Strategien. Zudem stimmen die Autoren darin überein, dass 
Unternehmen verschiedene Dienstleistungsstrategien gleichzeitig verfolgen können (Davies 2004; Windahl/Lakemond 2006). Die Artikel verwenden in der Regel das Dienstleistungsangebot zur Operationalisierung der Dienstleistungsstrategien. Eine Dienstleistungsstrategie beinhaltet ein ausgewähltes Dienstleistungsangebot und basiert auf dem damit verbundenen Wertversprechen. Eine Operationalisierung der Dienstleistungsstrategie über die angebotenen Dienstleistungen nutzt die bisherigen Beiträge nur unvollständig. Neben der Dimension der angebotenen Dienstleistungen empfiehlt sich die Operationalisierung einer Dienstleistungsstrategie über zwei weitere Dimensionen. Eine Dimension ist die Verbreitung des Dienstleistungsangebotes, welche angibt wie viele Kunden das Dienstleistungsangebot in Anspruch nehmen. Eine weitere Dimension ist die Intensität der Vermarktung des Dienstleistungsangebots. Wir empfehlen Dienstleistungsstrategien durch einen Index aus diesen drei Dimensionen zu operationalisieren (Homburg et al. 2002).

\subsection{Ergebnis der Faktorenanalyse}

Unsere erste Hauptkomponentenanalyse identifizierte Artikel mit Faktorladungen niedriger als 0,4. Diese Artikel wurden aus der weiteren Analyse eliminiert. Die finale Hauptkomponentenanalyse extrahierte sieben Faktoren (vgl. Tabelle 2). Diese Faktoren erklären 82,3 Prozent der Varianz. Ein Artikel mit einer Faktorladung grösser gleich 0,7 wurde als primärer Beitrag zu diesem Faktor bzw. der Forschungsrichtung interpretiert. Ein Artikel mit einer Faktorladung grösser gleich 0,4 und kleiner als 0,7 wurde als sekundärer Beitrag zu der Forschungsrichtung eingeordnet.

\begin{tabular}{|c|c|c|c|c|c|c|c|}
\hline & 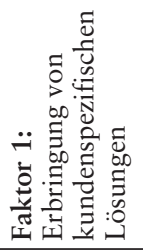 & 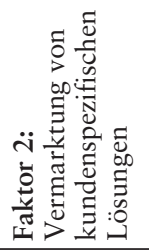 & 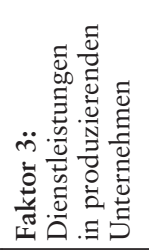 & 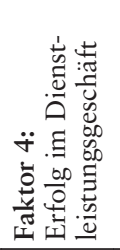 & 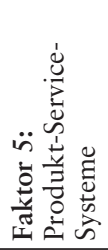 & 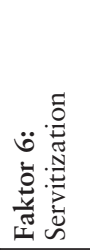 & 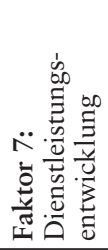 \\
\hline Davies (2004) & 0.92 & 0.42 & & & & & \\
\hline Galbraith (2002) & 0.83 & & & & & & \\
\hline Davies et al. (2006) & 0.87 & & & & & & \\
\hline Davies et al. (2007) & 0.86 & & & & & & \\
\hline Brady et al. (2005) & 0.76 & & & & & & \\
\hline Windabl/Lakemond (2006) & 0.81 & 0.56 & & & & & \\
\hline Wise/Baumgartner (1998) & 0.93 & & & & & & \\
\hline Sawhney et al. (2004) & 0.72 & & & & & & \\
\hline Matthyssens/Vandenbempt (2008) & 0.71 & & & & & & \\
\hline Tuli et al. (2007) & 0.59 & 0.81 & & & & & \\
\hline Cova/Salle (2008) & 0.47 & 0.80 & & & & & \\
\hline Pettinen/Palmer (2007) & 0.46 & 0.75 & & & & & \\
\hline Jacob/Ulaga (2008) & & & 0.81 & 0.44 & & & \\
\hline Vandenbempt/Matthyssen (1998) & & & 0.72 & & & & \\
\hline
\end{tabular}




\begin{tabular}{|c|c|c|c|c|c|c|c|}
\hline & 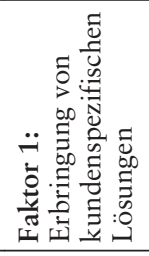 & 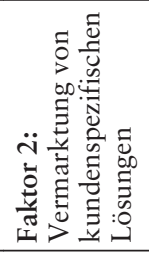 & 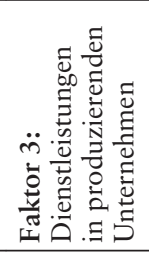 & 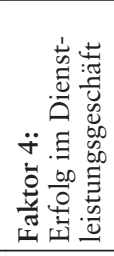 & 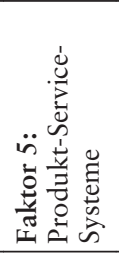 & 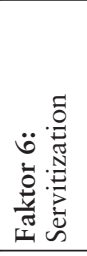 & 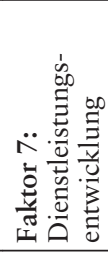 \\
\hline Oliva/Kallenberg (2003) & 0.41 & & 0.83 & 0.43 & & & \\
\hline Neu/Brown (2008) & & & 0.75 & & & & \\
\hline Gebauer (2008) & & & 0.81 & 0.41 & & & \\
\hline Mathieu (2001a) & & & 0.85 & 0.45 & & & \\
\hline Mathieu (2001b) & & & 0.70 & & & & \\
\hline Neu/Brown (2005) & & & 0.85 & 0.51 & & & \\
\hline Martin/Horne (1992) & & & 0.72 & & & & \\
\hline Gebaner et al. (2010) & & & 0.72 & & & & \\
\hline Fang et al. (2009) & & & & 0.84 & & & \\
\hline Gebauer et al. (2005) & & & 0.47 & 0.81 & & & \\
\hline Gebaner et al. (2006) & & & & 0.73 & & & \\
\hline Brax (2005) & & & & 0.73 & & & \\
\hline Antioco et al. (2008) & & & & 0.72 & & & \\
\hline Homburg et al. (2003) & & & & 0.72 & & & \\
\hline Tukker (2008) & & & & & 0.81 & & \\
\hline Roy (2000) & & & & & 0.78 & & \\
\hline Tukker/Tischner (2006) & & & & & 0.80 & & \\
\hline Markeset/Kumar (2003) & & & & & 0.71 & & \\
\hline Vandermerwe/Rada (1988) & & & & & & 0.43 & \\
\hline Baines et al. (2009) & & & & & & 0.51 & \\
\hline Neely (2008) & & & 0.54 & & & 0.43 & \\
\hline Brentani (1989) & & & & & & & 0.86 \\
\hline Brentani (1995) & & & & & & & 0.84 \\
\hline
\end{tabular}

Tabelle 2: Ergebnis der Faktorenanalyse

Bei der Benennung und Kategorisierung der Faktoren als Forschungsrichtungen berücksichtigten wir die gemeinsamen Inhalte der primären Beiträge. Sieben Forschungsrichtungen konnten dabei identifiziert werden: (1) Erbringung von kundenspezifischen Lösungen, (2) Vermarktung von kundenspezifischen Lösungen, (3) Dienstleistungen in produzierenden Unternehmen, (4) Erfolg im Dienstleistungsgeschäft, (5) Produkt-Service-Systeme, (6) Servitization und (7) Dienstleistungsentwicklung.

Faktor 1 zur Erbringung von kundenspezifischen Lösungen bildet eines von zwei strukturellen Zentren des Forschungsthemas. Die neun Artikel, die diesen Faktor bilden, gehen 
über das einfache Bündeln von Produkten und Dienstleistungen zu kundenspezifischen Lösungen hinaus. Der Fokus ist auf der Integration von Produkten und Dienstleistungen. Die Artikel diskutieren die Fähigkeiten und Strukturen zur Definition, Vermarktung und Erbringung von Lösungen. Diese Fähigkeiten und Strukturen fördern eine stärkere Kundenorientierung. Die Artikel beschreiben keine Sequenz im Ausbau des Lösungsgeschäfts, sondern konzentrieren sich auf das Wertversprechen und die Wertgenerierung.

Faktor 2 umfasst drei Artikel über die Vermarktung von kundenspezifischen Lösungen und ergänzt den Faktor 1. Diese Artikel diskutieren wie Lösungen die Marketingaktivitäten beeinflussen. Auf einer konzeptionellen Ebene wandeln sich Marketingaktivitäten von einer Transaktions- zu einer Beziehungsorientierung. Auf der operativen Ebene gehen Marketingkompetenzen über das Management der Kundenbeziehung hinaus. Marketingkompetenzen beinhalten Fähigkeiten zum Verständnis der Kundenbedürfnisse, der Anpassung der Lösungen an die Kundenbedürfnisse und der Integration der Produkte und Dienstleistungen bei den Lösungen.

Faktor 3 beinhaltet Dienstleistungen in produzierenden Unternehmen. Faktor 3 ist das zweite strukturelle Zentrum. Es laden zehn Artikel auf diesen Faktor. Diese Artikel zeigen folgende Gemeinsamkeiten und Unterschiede zu Faktor 1 auf. Die Artikel betonen einzelne Dienstleistungskategorien und nicht die Integration von Produkten und Dienstleistungen. Es geht um die Bündelung von Produkten mit einzelnen Dienstleistungskategorien (Martin/ Horne 1992). Die Artikel konzentrieren sich auf die Beschreibung der Dienstleistungskategorien und beschäftigen sich nur nebensächlich mit dem Wertversprechen dieser Kategorien. Der Ausbau des Dienstleistungsangebots folgt einer Sequenz beginnend mit Basisdienstleistungen und fortgeführt durch komplexere und wissensintensivere Dienstleistungen. Beim Faktor 1 gibt es ein gemeinsames Verständnis von Lösungen, während die Artikel in Faktor 3 verschiedene Dienstleistungskategorien diskutieren (z.B. Kundendienst, After-Sales Services, operative Dienstleistungen, produkt- und kundenunterstützende Dienstleistungen). Faktor 3 betont die Dienstleistungsorientierung und nicht die Kundenorientierung. Faktor 1 beschreibt die Ausgestaltung der Verkaufsteams, Kompetenzen im Kundenkontakt und Organisationsstruktur zur Erhöhung der Kundenorientierung (Galbraith 2002; Davies et al. 2006; Davies et al. 2007). Faktor 3 beinhaltet die Fähigkeiten und Organisationsstrukturen für eine höhere Dienstleistungsorientierung. Die Artikel diskutieren beispielsweise ob die Etablierung eines separaten Geschäftsbereiches für Dienstleistungen oder die Integration von Dienstleistungen und Produkten in einen Geschäftsbereich die Dienstleistungsorientierung fördern (Oliva/Kallenberg 2003; Neu/Brown 2005; Gebauer et al. 2010). Bei den Fähigkeiten werden dienstleistungsorientierte Verhaltensweisen und Mitarbeiterkompetenzen diskutiert (Edvardsson et al. 2008).

Faktor 4 zum Erfolg im Dienstleistungsgeschäft ergänzt Faktor 3 mit finanziellen und nicht-finanziellen Kennzahlen wie den Beitrag der Dienstleistungen zum Umsatz, Gewinn und Kundenzufriedenheit und die dafür notwendigen Investitionen (Gebauer/Fleisch 2007; Antioco et al. 2008). Während der Umsatzanteil von Dienstleistungen in jedem der Artikel bei Faktor 4 enthalten ist, werden die Dienstleistungsprofitabilität, nicht-finanzielle Kennzahlen wie Kundenloyalität und -zufriedenheit (Homburg et al. 2003) sowie Investitionen (Gebauer et al. 2005; Gebauer/Fleisch 2007) nur in wenigen Artikeln beschrieben.

Faktor 5 beinhaltet vier Artikel. Die Artikel führen Tukker's (2004) Produkt-ServiceSysteme fort und zeigen auf wie diese Produkt-Service-Systeme zu implementieren sind. Fragestellungen wie beispielsweise, welche Organisationsstrukturen und Fähigkeiten für 
die Implementierung eines ausgewählten Produkte-Service-Systems konkret notwendig sind, werden jedoch nicht beantwortet.

Faktor 6 zu Servitization beinhaltet drei Artikel. Sie diskutieren die operativen Strategien, Fähigkeiten und Organisationsstrukturen für einen erfolgreichen Servitizationprozess in produzierenden Unternehmen.

Faktor 7 beinhaltet zwei Artikel zum Thema Dienstleistungsentwicklung.

\subsection{Ergebnis der MDS}

Die MDS ergänzt die Faktorenanalyse (vgl. Abbildung 2). Die horizontale Achse entspricht der Wertschöpfungskette. Am linken Ende der Achse befinden sich Artikel, die sich mit den vorgelagerten (upstream) Aktivitäten in der Wertschöpfungskette beschäftigen. Diese Artikel betrachten den Wandel vom Produzenten zum produzierendem Dienstleister aus der Perspektive des Unternehmens und dessen Zulieferunternehmen. Davies et al.'s (2007) Unterscheidung in Systemintegrator und Systemlieferant ist beispielsweise auf der linken Seite positioniert. Systemintegratoren haben nur einen Teil der Fähigkeiten zur Erbringung der kundenspezifischen Lösungen selbst. Für weitere Fähigkeiten kooperieren Systemintegratoren mit Zulieferunternehmen. Ein Systemlieferant verfügt selbst über alle Fähigkeiten zur Erbringung der kundenspezifischen Lösungen. Am rechten Ende der Achse sind Artikel, welche sich mit nachgelagerten (downstream) Aktivitäten beschäftigen. Diese Artikel betrachten den Wandel aus Kundenperspektive. Inhalte der Artikel sind Wertversprechen oder Beziehungsprozesse zwischen produzierenden Unternehmen und Kunden. Typische Beispiele sind die von Tuli et al. (2007) beschriebenen Beziehungsprozesse zwischen Unternehmen und Kunden. Die Mehrzahl der Artikel gruppiert sich um den mittleren Teil der horizontalen Achse. Diese Artikel betrachten das Unternehmen selbst, welches sich schrittweise vom Produzenten zum produzierenden Dienstleister weiterentwickelt. Neu und Brown (2005) diskutieren beispielsweise die notwendigen Veränderungen in der Strategie und den organisationalen Faktoren (Prozesse, Kompetenzen, Strukturen und Anreizsysteme).

Die vertikale Achse kann als Kontinuum zwischen Artikeln mit strategischem oder operativem Fokus interpretiert werden. Artikel am oberen Ende der vertikalen Achse haben einen strategischen Fokus. Sie beschreiben verschiedene Dienstleistungsstrategien (Mathieu 2001a; Gebauer 2008; Windahl/Lakemond 2010). Am unteren Ende der vertikalen Achse sind Artikel mit einem operativen Fokus. Diese Artikel beschäftigen sich mit den individuellen Verhaltensmustern von Mitarbeitern (Gebauer/Friedli 2005), Dienstleistungsentwicklungsprozessen (Brentani 1989) oder der Preissetzung von Dienstleistungen (Malleret 2006). Die meisten Artikel gruppieren sich um den mittleren Teil der vertikalen Achse und können auf einer taktischen Ebene eingeordnet werden. Hierzu zählen Artikel, welche die Dienstleistungsorientierung in den organisationalen Strukturen oder der Unternehmenskultur diskutieren (z.B. Homburg et al. 2003; Neu/Brown 2005). 


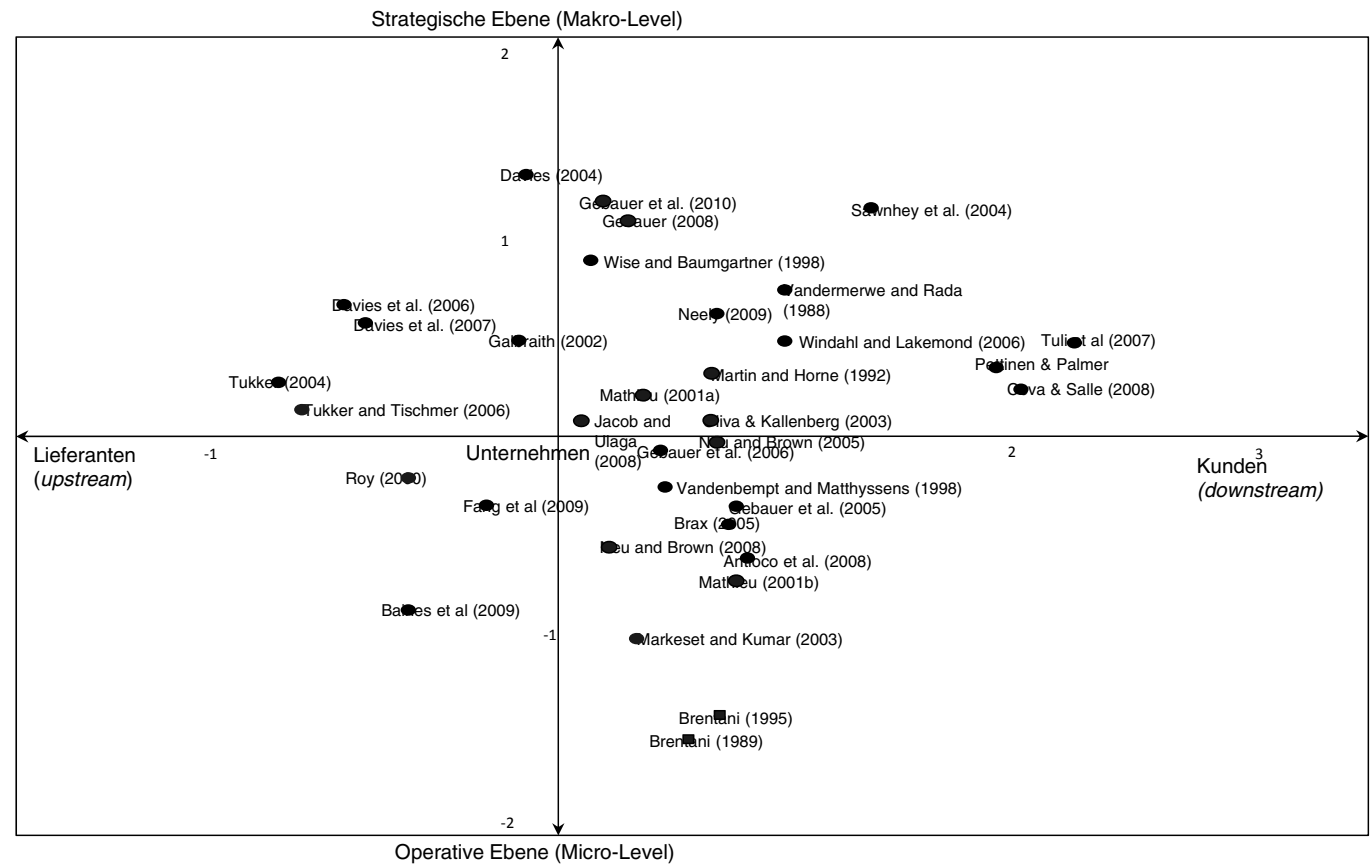

Abbildung 2: Grafische Konfiguration der MDS

\section{Diskussion der Handlungsempfehlungen}

Aus den Resultaten unserer bibliometrischen Analyse lassen sich empirische und theoretische Handlungsempfehlungen für die Weiterentwicklung des Forschungsthemas ableiten.

\subsection{Empirische Handlungsempfehlungen}

Empirische Handlungsempfehlungen beginnen mit der Verwendung von Longitudinalstudien und der Konzentration auf nichterfolgreiche Initiativen zum Ausbau des Dienstleistungsgeschäfts. Die aufgenommenen Fallstudien sind von Unternehmen, die erfolgreich beim Ausbau des Dienstleistungsgeschäfts sind. Gleichzeitig wird darauf verwiesen, dass eine Mehrzahl der Unternehmen Schwierigkeiten beim Ausbau des Dienstleistungsgeschäfts haben. Schwierigkeiten entsprechen dem Service Paradox oder einem fehlerhaften Ausbau des Dienstleistungsgeschäfts. Dadurch entsteht eine Verzerrung der Resultate. Fehler beim Ausbau des Dienstleistungsgeschäft werden selten aufgezeigt und es bleibt unklar, ob die Steigerungen im Dienstleistungsumsatz und -gewinn den Erwartungen entsprechen oder nicht. Dadurch entsteht ein zu positiver Blick auf die Transformation vom Produkt zur Dienstleistung. Probleme beim Ausbau des Dienstleistungsgeschäfts und deren Ursachen wie Managementfehler oder Fehlinvestitionen bleiben unentdeckt. Natürlich sind Unternehmen nicht daran interessiert ihre Fehler zu veröffentlichen und es ist schwierig die Daten aufzunehmen. Trotzdem ist die Analyse dieser Fehler entscheidend für die Theoriebildung. Forscher müssen Möglichkeiten finden, mit diesen Unternehmen zusammenzuarbeiten, ihre Daten aufzunehmen und zu analysieren. Eine weitere Möglichkeit sind komparative Studien, die Unternehmen vergleichen, die einen hohen und niedrigen Anteil vom 
Dienstleistungsumsatz am Unternehmensumsatz haben oder deren Erwartungen an den Ausbau des Dienstleistungsgeschäfts erfüllt bzw. nicht erfüllt wurden.

Longitudinalstudien sind konsistent mit der Erkenntnis, dass der Wandel vom Produzenten zum produzierenden Dienstleister ein langfristiger und inkrementeller Prozess ist (Kowalkowski et al. 2012). Zudem wirken Verbesserungen im Dienstleistungsbereich (z.B. Entwicklung neuer Dienstleistungen, der Aufbau eines strategischen Geschäftsbereichs für Dienstleistungen, zunehmende Dienstleistungsorientierung in der Unternehmenskultur oder im Personalmanagement) verzögert auf den Dienstleistungsumsatz und -gewinn aus. Zukünftige Studien sollten empirische Daten nicht wie bisher zu einem bestimmten Zeitpunkt erheben, sondern einen Zeitraum von mindestens fünf Jahren betrachten. Über diesen Zeitraum hinweg müssen Kennzahlen für den zunehmenden Beitrag von Dienstleistungen zum Unternehmenserfolg analysiert werden.

Die Beschreibung des Umsatzanteils, der durch Dienstleistungen erwirtschaftet wird, sollte als Qualitätsindikator für empirische Studien etabliert werden. Hierzu ist es notwendig, die Art und Weise der Berechnung des Dienstleistungsumsatzes offenzulegen. Dabei ist zu berücksichtigen, ob der Ersatzteilumsatz als Dienstleistungs- oder Produktumsatz ausgewiesen wird und ob die Dienstleistungen durch eigene Marktorganisationen oder unabhängige Vertretungen erbracht werden.

Die hohe Profitabilität und der hohe Umsatzanteil von Ersatzteilen erfordert eine genaue Zuordnung. Marktorganisationen erwirtschaften internen Dienstleistungsumsatz und Unternehmen weisen diesen Umsatz in der Regel aus. Wenn unabhängige Vertretungen die Dienstleistungen erbringen, dann handelt es sich um externen Dienstleistungsumsatz. Der externe Dienstleistungsumsatz wird nicht ausgewiesen. Grosse multinationale Unternehmen verfügen ausschliesslich über Marktorganisationen. KMUs bearbeiten den Markt tendenziell durch unabhängige Vertretungen. Mittelständische Unternehmen haben eigene Marktorganisationen in den wichtigen Märkten und unabhängige Vertretungen in den weniger wichtigen Märkten. Eine Kennzahl wie Anteil von Dienstleistungen am Gesamtumsatz von 24 Prozent ist nur aussagekräftig, wenn nachvollziehbar ist, ob neben dem internen weiterer externer Dienstleistungsumsatz erwirtschaftet wird.

Das Verhältnis zwischen Neuproduktgeschäft und installierter Basis beeinflusst ebenfalls den Umsatzanteil für Dienstleistungen. Je kleiner das Verhältnis ist, desto höher ist der Umsatzanteil. In diesem Fall verkaufen Unternehmen nur relativ wenige Neuprodukte, sie verfügen jedoch über sehr viele installierte Produkte für die Dienstleistungen vermarkten werden können. Ähnlich ist es mit dem Verhältnis von Investitions- und Lebenszykluskosten. Hohe Lebenszykluskosten bedeuten hohe Ausgaben für Ersatzteile, Reparaturen und Wartungen. D.h. je kleiner dieses Verhältnis, desto höher ist der Dienstleistungsumsatz. Eine Interpretation des Anteils von Dienstleistungen am Gesamtumsatz ist deswegen nur möglich, wenn diese Verhältnisse ebenfalls beschrieben sind.

Der Umsatzanteil von Dienstleistungen sollte nach Möglichkeit mit zwei Indikatoren ergänzt werden. Der erste Indikator ist die Detaillierung des Dienstleistungsumsatzes hinsichtlich einzelner Dienstleistungskategorien. Diese Detaillierung geht über die konkrete Ausweisung des Ersatzteilumsatzes hinaus. Sie dient der Konkretisierung einzelner Dienstleistungsstrategien. Bisher werden Dienstleistungsstrategien durch das Dienstleistungsangebot operationalisiert. Die Strategie eines Entwicklungspartners basiert auf dem Angebot von Dienstleistungen wie Machbarkeitsstudien, Forschungs- und Entwicklungsdienstleistungen oder Konstruktionsdienstleistungen. Um die strategische Positionierung als Ent- 
wicklungspartner zu validieren, ist eine Darstellung des Umsatzanteils für diese Dienstleistungen notwendig. Die Dienstleistungsprofitabilität ist der zweite Indikator. Unternehmen veröffentlichen diese Daten zunehmend in ihren Jahresberichten. Eine Dienstleistungsorganisation, die ein separater strategischer Geschäftsbereich ist, weist Umsatz und Profitabilität aus.

Neben dem Anteil von Dienstleistungen am Gesamtumsatz lässt sich der Wandel vom Produzenten zum produzierenden Dienstleister auch durch nicht-finanzielle Indikatoren wie dem Umfang und der Intensität des Dienstleistungsangebots erfassen. Umfang und Intensität setzen die Dienstleistungen in Bezug zu den Kundenaktivitäten. Der Umfang entspricht dem Anteil der Kundenaktivitäten entlang der Vorkauf-, Kauf- und Nutzungsphase, welche durch Dienstleistungen unterstützt werden. Intensität bedeutet wie ausgeprägt die Unterstützung in den Aktivitäten ist. Umfang und Intensität bilden einen Indikator für den Grad der Unterstützung der Kundenaktivitäten mit Hilfe von Dienstleistungen.

\subsection{Theoretische Handlungsempfehlungen}

Theoretische Handlungsempfehlungen lassen sich aus der Faktoranalyse und der MDS ableiten. Wir empfehlen eine verstärkte Ausnutzung der Synergien zwischen den sieben Forschungsrichtungen. Dies beginnt mit einer besseren Integration der Konzepte Dienstleistungs- und Kundenorientierung, welche einen wichtigen Teil der strukturellen Zentren Erbringung von kundenspezifischen Lösungen und Dienstleistungen in produzierenden Unternehmen bilden. Die Forschungsrichtungen Erbringung von kundenspezifischen Lösungen und Vermarktung von kundenspezifischen Lösungen sollten verstärkt die finanziellen und nicht-finanziellen Erfolgskennzahlen der Forschungsrichtung Erfolg im Dienstleistungsgeschäft nutzen. Artikel über den Aufbau des Lösungsgeschäfts sollten Auskunft über den Anteil des Lösungsgeschäfts am Gesamtumsatz geben. Für die Forschungsrichtung Dienstleistungen in produzierenden Unternehmen empfehlen wir eine stärkere Fokussierung auf die Integration von Produkten und Dienstleistungen. Anstatt der Betrachtung wie beispielsweise Produkte und Outsourcingdienstleistung miteinander gebündelt werden, empfehlen wir zu untersuchen wie Outsourcingdienstleistungen die Entwicklung neuer Produkte beeinflussen.

Aus der MDS lassen sich folgende Handlungsempfehlungen für die theoretische Weiterentwicklung ableiten. Diese Handlungsempfehlungen resultieren aus den mit wenigen Artikeln besetzten Bereichen in der grafischen Konfiguration der MDS. Wir empfehlen eine stärkere Mikro-Fundierung der operativen Themen wie Dienstleistungsinnovationsprozesse, Mitarbeiterkompetenzen, Verhaltensmuster oder Preisgestaltung von Dienstleistungen. Diese Mikro-Fundierung betont insbesondere die Interaktionen zwischen Unternehmen und Kunden oder zwischen Unternehmen und Lieferanten. Mögliche Forschungsfragen sind: Wie können Kunden oder Lieferanten in den Dienstleistungsinnovationsprozess einbezogen werden?, Wie entstehen dienstleistungsorientierte Kompetenzen und Verhaltensweisen in der Zusammenarbeit zwischen Unternehmen, Lieferanten und Kunden? oder Wie kann die Einbindung von Kunden und Lieferanten zu einer optimalen Preisgestaltung für Dienstleistungen führen?

Die Makro-Fundierung auf der strategischen Ebene ist ein Aufruf für eine intensivere Diskussion der strategischen Implikationen. Eine interessante Forschungsfrage wäre die Rolle von Mergern \& Akquisitionen (M\&As). Die bisherigen empirischen Studien gehen davon aus, dass der Ausbau des Dienstleistungsgeschäfts auf internem Wachstum und ei- 
gener Kompetenzentwicklung basieren. Unternehmen können ebenfalls das Dienstleistungsgeschäft durch externes Wachstums ausbauen. Anstatt in die stärkere Dienstleistungsorientierung der eigenen Kompetenzen, Prozesse und Strukturen zu investieren, können Unternehmen diese auch extern akquirieren. Aus Unternehmenssicht kann es ökonomisch sinnvoll sein, spezialisierte Dienstleistungsunternehmen zu akquirieren. Die Betrachtung von M\&As betrifft nicht nur spezialisierte Dienstleister. Ebenfalls interessant sind M\&As mit anderen Herstellern von Produkten. Die strategischen Argumente sind hier die Erhöhung und Verbreiterung der installierten Basis. Erhöhung bedeutet beispielsweise, dass ein Hersteller von Verpackungsmaschinen einen anderen Verpackungsmaschinenhersteller aufkauft. Die Anzahl der installierten Verpackungsmaschinen, für die Dienstleistungen vermarktet werden können, steigt. Unternehmen können Effizienzgewinne in der Dienstleistungsorganisation erzielen. Verbreiterung der installierten Basis bedeutet, dass ein Verpackungsmaschinenhersteller weitere Hersteller von Maschinen und Anlagen (z.B. Automatisierungstechnik, Roboter usw.) im Verpackungsprozess akquiriert. Das Unternehmen weitet das Dienstleistungsangebot auf diese Maschinen und Anlagen aus und kann damit mehr Kundenaktivitäten und -bedürfnisse abdecken. Neben den M\&As ist die Untersuchung strategischer Allianzen ein wichtiger Beitrag zur Makro-Fundierung des Forschungsthemas.

Eine weitere Makro-Fundierung wäre ein besseres Verständnis wie Dienstleistungen nachhaltige Wettbewerbsvorteile generieren. Mögliche Forschungsfragen sind: Wie können Unternehmen einen direkten Wettbewerbsvorteil durch ihre Servicekompetenzen erzeugen? Wie können Unternehmen Servicekompetenzen zu Kernkompetenzen weiterentwickeln? Welche Massnahmen müssen Unternehmen treffen, damit Servicekompetenzen nicht einfach von Wettbewerben imitiert werden? Ulaga und Reinartz (2012) argumentieren, dass nachhaltigen Wettbewerbsvorteile nicht aus Servicekompetenzen entstehen, sondern aus einer Kombination aus Produkt- und Dienstleistungskompetenzen. Zum besseren Verständnis wie Dienstleistungskompetenzen Wettbewerbsvorteile generieren, empfiehlt es sich Dienstleistungen nicht isoliert zu betrachten. Dienstleistungskompetenzen und die daraus resultierende Dienstleistungsdifferenzierung sollten mit anderen Faktoren der Wettbewerbsdifferenzierung kombiniert werden (z.B. Markt- und Kundenorientierung, Innovationsfähigkeit oder Produktdifferenzierung) (Gebauer et al. 2011).

Die Forschungsfragen betreffen die vor- und nachgelagerten Wertschöpfungsaktivitäten. Bei den Richtung Kunden vorgelagerten Aktivitäten ist zu berücksichtigen, dass Servicekompetenzen durch eine Ko-Kreation mit Kunden entstehen. Kunden nehmen dadurch einen Einfluss auf den Aufbau von Servicekompetenzen. Auf der einen Seite können durch diese auf einzelne Kunden spezialisierten Servicekompetenzen Eintrittsbarrieren für Wettbewerber entstehen. Auf der anderen Seite sind diese kundenspezifischen Servicekompetenzen nur schwer auf weitere Kunden anzuwenden. Kunden stehen im Wettbewerb. Sie haben wenig Interesse, eine für sie entwickelte Lösung auf andere Kunden zu übertragen. Unter der Berücksichtigung von Christensens' Innovationsdilemma (Christensen et al. 2002), Ko-Kreation mit Kunden führt zur zunehmende Spezialisierung der Kompetenzentwicklung. Sie kann jedoch nachteilig für die Fähigkeit zur Entwicklung strategischer Innovationen sein. Strategische Innovationen ermöglichen neue Wertkonstellationen und eröffnen radikal neue Wege zur Befriedung der Kundenbedürfnisse. Kunden honorieren derartige strategischen Innovationen nicht unbedingt und favorisieren eher inkrementelle Verbesserungen (Christensen et al. 2002; Matthyssens/Vandendempt 2010). Zukünftige For- 
schungsfragen können beantworten, ob die Transformation vom Produkt zur Dienstleistung Unternehmen vor disruptiven Technologien exponiert und möglicherweise strategische Innovationen erschwert.

Einen interessanten theoretischen Beitrag könnte ebenfalls der Ansatz zur Boundary of the Firm leisten. Der Wandel vom Produkt zur Dienstleistung erweitert die Grenze des Unternehmens. Unternehmen weiten ihre Wertschöpfungsaktivitäten aus und verbreitern die Kompetenzen, in dem sie die notwendigen Kompetenzen für das Dienstleistungsgeschäft aufbauen. Diese Erweiterung der Kompetenzen ist gegenläufig zu dem Argument der stärkeren Spezialisierung und Fokussierung auf wenige Kernkompetenzen sowie der damit verbundenen Reduktion der Kompetenzen und der Grenze des Unternehmens. Die Untersuchung des Wandels vom Produzenten zum produzierenden Dienstleister kann einen wichtigen Beitrag zur Diskussion um die Grenze des Unternehmens und der Spezialisierung auf Kernkompetenzen leisten.

\section{Zusammenfassung}

In diesem Artikel untersuchten wir die Entwicklung des schnell wachsenden Forschungsthemas über die Transformation vom Produzenten zum produzierenden Dienstleister. Wir artikulieren einige der kritischen empirischen und theoretischen Bedenken. Wir verwenden eine bibliometrische Analyse, um unser Ziel zu erreichen. Diese Analyse hat Limitationen. Zitationen sind beispielsweise nur ein verzögerter Indikator für den Einfluss eines Artikels. Es ist zudem schwierig aus einer bibliometrischen Analyse zukünftige Forschungsthemen abzuleiten (Brown/Gardner 1985). Ausserdem ignoriert die Methode die Rolle von Büchern (z.B. Belz et al. 1997).

Dennoch trägt das Aufdecken der Strukturen zur weiteren Entwicklung des Themas bei. Die Identifikation des intellektuellen Kerns, die Phasen für die Entwicklung des Themas usw. verbessern das Verständnis für das Forschungsthema und für die bestehenden und zukünftigen Forschungsrichtungen. Forschung kann von der Ausnutzung der Synergien und der Kombination der Forschungsrichtungen (z.B. Erbringen von Lösungen, Vermarktung von Lösungen, Dienstleistungen in produzierenden Unternehmen, Erfolg im Dienstleistungsgeschäft, Produkt-Service-Systeme und Dienstleistungsentwicklung) profitieren. Aufgrund der ersten Reifephase des Forschungsthemas ist es wichtig, die zukünftigen Forschungsaktivitäten auszurichten. Unsere Ergebnisse zeigen konkrete Wege zur Erweiterung und Vertiefung der empirischen und theoretischen Forschung auf.

\section{Literaturhinweise}

Allmendinger, G./Lombreglia, R. (2005): Four strategies for the age of smart services, in: Harvard Business Review, Jg. 83, Nr. 10, S. 131-145.

Antioco, M./Moenaert, R.K./Lindgreen, A./Wetzels, M.G. (2008): Organizational antecedents to and consequences of service business orientations in manufacturing companies, in: Journal of the Academy of Marketing Science, Jg. 36, Nr. 3, S. 337-358.

Araujo, L./Spring, M. (2006): Services, products, and the institutional structure of production, in: Industrial Marketing Management, Jg. 35, Nr. 7, S. 797-805.

Backhaus, K. (2003): Industriegütermarketing, 7. Auflage, München. 
Baines, T.S./Lightfoot, H.W./Benedettini, O./Kay, J.M. (2009): The servitization of manufacturing: a review of literature and reflection on future challenges, in: Journal of Manufacturing Technology Management, Jg. 20, Nr. 5, S. 547-67.

Belz, C./Schuh, G./Groos, A./Reinecke, S. (1997): Industrie als Dienstleister, St. Gallen: Verlag Thexis.

Bowen, D.E./Siehl, C./Schneider, B. (1989): A Framework for Analyzing Customer Service Orientations in Manufacturing, in: Academy of Management Review, Jg. 14, Nr. 1, S. 75-95.

Boyt, T./Harvey, M. (1997): Classification of Industrial Services: A Model with Strategic Implications, in: Industrial Marketing Management, Jg. 26, Nr. 4, S. 291-300.

Brady, T./Davies, A./Gann, D. (2005): Creating value by delivering integrated solutions, in: International Journal of Project Management, Jg. 23, Nr. 3, S. 360-365.

Brax, S. (2005): A manufacturer becoming service provider - challenges and a paradox, in: Managing Service Quality, Jg. 15, Nr. 2, S. 142-155.

Brentani de, U. (1989): Success and Failure in New Industrial Services, in: Journal of Product Innovation Management, Jg. 6, Nr. 4, S. 239-258.

Brentani de, U. (1995): New industrial service development: Scenarios for success and failure, in: Journal of Business Research, Jg. 32, Nr. 2, S. 93-103.

Brown, L.D./Gardner, J.C. (1985): Using citation analysis to assess the impact of journals and articles on contemporary accounting research (CAR), in: Journal of Accounting Research, Jg. 23, Nr. 1, S. 84-109.

Christensen, C.M./Johnson, M.W./Rigby, D.K. (2002): Foundations for growth. How to identify and build disruptive new businesses, in: Sloan Management Review, Jg. 43, Nr. 3, S. 22-31.

Cohen, M./Agrawal, N./Agrawal, V. (2006): Winning in the Aftermarket, in: Harvard Business Review, Jg. 85, Nr. 4, S. 129-138.

Cova, B.,/Salle, R. (2008): Marketing solutions in accordance with the SD logic: Co-creating value with customer network actors, in: Industrial Marketing Management, Jg. 37, Nr. 3, S. 270-277.

Culnan, M. (1986): The intellectual development of management information systems, 1972-1982: A co-citation analysis, in: Management Science, Jg. 32, Nr. 2, S. 156-72.

Davies, A. (2004): Moving base into high-value integrated solutions: a value stream approach, in: Industrial and Corporate Change, Jg. 13, Nr. 5, S. 727-756.

Davies, A./Brady, T.,/Hobday, M. (2006): Charting a Path Toward Integrated Solutions, in: MIT Sloan Management Review, Jg. 47, Nr. 3, S. 39-48.

Davies, A.,/Brady, T./Hobday, M. (2007): Organizing for solutions: Systems seller vs. systems integrator, in: Industrial Marketing Management, Jg. 36, Nr. 2, S. 183-193.

Droege, H./Hildebrandt, D./Heras Forcada, M. A. (2009): Innovation in services: present findings, and future pathways, in: Journal of Service Management, Jg. 20, Nr. 2, S. 131-55.

Edvardsson, B./Holmlund, M.,/Strandvik, T. (2008): Initiation of business relationships in servicedominant settings, in: Industrial Marketing Management, Jg. 37, Nr. 3, S. 339-350.

Fang, E./Palmatier, R./Steenkamp, J. (2008): Effect of service transition strategies on firm value, in: Journal of Marketing, Jg. 72, Nr. 1, S. 1-14.

Galbraith, J.R. (2002): Organizing to deliver solutions, in: Organizational Dynamics, Jg. 31, Nr. 2, S. 194-207.

Gebauer, H. (2008): Identifying service strategies in product manufacturing companies by exploring environment-strategy configurations, in: Industrial Marketing Management, Jg. 37, Nr. 3, S. 278-91. 
Gebauer, H./Edvardsson, B./Gustafsson, A./Witell, L. (2010): Match or mismatch: strategy-structure configurations in the service business of manufacturing companies, in: Journal of Service Research, Jg. 13, Nr. 2, S. 198-215.

Gebauer, H./Fleisch, E. (2007): An investigation of the relationship between behavioral processes, motivation, investments in the service susiness and Service Revenue, in: Industrial Marketing Management, Jg. 36, Nr. 3, S. 337-348.

Gebauer, H./Fleisch, E./Friedli, T. (2005): Overcoming the service paradox in manufacturing companies, in: European Management Journal, Jg. 23, Nr. 1, S. 14-26.

Gebauer, H./Friedli, T. (2005): Behavioral implications of the transition process from products to services, in: Journal of Business \& Industrial Marketing, Jg. 20, Nr. 2, S. 70-78.

Gebauer, H./Friedli, T./Fleisch, E. (2006): Success factor for achieving high service revenues in manufacturing companies, in: Benchmarking: An international Journal, Jg. 13, Nr. 3, S. 374-386.

Gebauer, H./Gustafsson, A./Witell, L. (2011): Competitive advantage through service differentiation by manufacturing companies, in: Journal of Business Research, Jg. 64, Nr. 12, S. 1270-1280.

Homburg, C./Fassnacht, M./Günther, C. (2003): The Role of Soft Factors in Implementing a Service-Oriented Strategy in Industrial Marketing Companies, in: Journal of Business-to-Business Marketing, Jg. 10, Nr. 2, S. 23-51.

Homburg, C./Hoyer, W. D./Fassnacht, M. (2002): Service Orientation of a Retailer's Business Strategy: Dimensions, Antecedents, and Performance Outcomes, in: Journal of Marketing, Jg. 66, Nr. 4, S. 86-101.

Jackson, R.W./Neidell, L.A./Lunsford, D. A. (1995): An empirical investigation of the differences in goods and services as perceived by organizational buyers, in: Industrial Marketing Management, Jg. 24, Nr. 2, S. 99-108.

Jackson, R.W./Cooper, P.D. (1988): Unique aspects of marketing industrial services, in: Industrial Marketing Management, Jg. 17, Nr. 2, S. 111-118.

Jacob, F./Ulaga, W. (2008): The transition from product to service in business markets: An agenda for academic inquiry, in: Industrial Marketing Management, Jg. 37, Nr. 3, S. 247-253.

Johansson, P./Olhager, J. (2004): Industrial service profiling: Matching service offerings and processes, in: International Journal of Production Economics, Jg. 89, Nr. 3, S. 309-320.

Kindstrom, D. (2010): Towards a service-based business model - Key aspects for future competitive advantage, in: European Management Journal, Jg. 28, Nr. 6, S. 479-490.

Kowalkowski, C./Kindstrom, D./Alejandro, T.B./Brege, S./Biggemann, S. (2012): Service infusion as agile incrementalism in action, in: Journal of Business Research, Jg. 65, Nr. 6, S. 765-772.

Kunz, W.H./Hogreve, J. (2011): Toward a deeper understanding of service marketing: the past, the present, and the future, in: International Journal of Research in Marketing, Jg. 28, Nr. 4, S. 231-247.

Leydesdorff, L. (2005): Similarity measures, author cocitation analysis, and information theory, in: Journal of the American Society for Information Science and Technology, Jg. 56, Nr. 7, S. 769-772.

Malleret, V. (2006): Value Creation through Service Offers, in: European Management Journal, Jg. 24, Nr. 1, S. 106-116.

Markeset, T./Kumar, U. (2005): Product support strategy: Conventional versus functional products, in: Journal of Quality in Maintenance Engineering, Jg. 11, Nr. 1, S. 53-67.

Martin, C.R./Horne, D.A. (1992): Restructuring towards a service orientation: the strategic challenges, in: International Journal of Service Industry Management, Jg. 3, Nr. 1, S. 25-38. 
Mathieu, V. (2001a): Service strategies within the manufacturing sector: benefits, costs and partnership, in: International Journal of Service Industry Management, Jg. 12, Nr. 5, S. 451-475.

Mathieu, V. (2001b): Product services: from a service supporting the product to a service supporting the client, in: Journal of Business and Industrial Marketing, Jg. 16, Nr. 1, S. 39-58.

Matthyssens, P./Vandenbempt K. (1998): Creating competition advantage in industrial services, in: Journal of Business \& Industrial Marketing, Jg. 13, Nr. 4/5, S. 339-355.

Matthyssens, P./Vandenbempt, K. (2008): Moving from basic offerings to value-added solutions: Strategies, barriers and alignment, in: Industrial Marketing Management, Jg. 37, Nr. 3, S. 316-328.

McCain, K.W. (1990): Mapping authors in intellectual space: a technical overview, in: Journal of the American Society for Information Science, Jg. 41, Nr. 6, S. 433-443.

Neely, A. (2008): Exploring the financial consequences of the servitization of manufacturing, in: Operations Management Research, Jg. 1, Nr. 2, S. 103-118.

Nerur, S./Rasheed, A./Natarajan, V. (2008): The intellectual structure of the strategic management field: an author co-citation analysis, in: Strategic Management Journal, Jg. 29, S. 319-336.

Neu, W./Brown, S. (2005): Forming Successful Business-to-Business Services in Goods-Dominant Firms, in: Journal of Service Research, Jg. 8, Nr. 1, S. 3-17.

Neu, W./Brown, S. (2008): Manufacturers forming successful complex business serv-ices: Designing an organization to fit the market, in: International Journal of Service Industry Management, Jg. 19 , Nr. 2, S. 232-251.

Oliva, R./Kallenberg, R. (2003): Managing the transition from products to services, in: International Journal of Service Industry Management, Jg. 14, Nr. 2, S. 160-172.

Ostrom, A./Bitner, M.J./Brown, S./Burkhard, K./Goul, M./Smith-Daniels, V./Demirkan, H./Rabinovich, E. (2010): Moving Forward and Making a Difference: Research Priorities for the Science of Service, in: Journal of Service Research, Jg. 13, Nr. 1, S. 4-36.

Penttinen, E./Palmer, J. (2007): Improving firm positioning through enhanced offerings an buyerseller relationships, in: Industrial Marketing Management, Jg. 36, Nr. 5, S. 552-564.

Perreault W./Leigh, L. (1989): Reliability of nominal data based on qualitative judgments, in: Journal of Marketing Research, Jg. 26, Nr. 2, S. 135-148.

Quinn, J.B./Doorley, T.L./Paquette, P.C. (1990): Beyond products: services-based strategy, in: Harvard Business Review, Jg. 68, Nr. 2, S. 58-67.

Roy, R. (2000): Sustainable product-service systems, in: Futures, Jg. 32 Nr. 3, S. 289-299.

Sawhney, M./Balasubramanian, S./Krishnan, V. V. (2004): Creating growth with sevices, in: Sloan Management Review, Jg. 34, Nr. 4, S. 34-43

Slack, N./Lewis, M./Bates, H. (2004): The two worlds of operations management research and practice: can they meet, should they meet?, in: International Journal of Operations \& Production Management, Jg. 24, Nr. 4, S. 372-387.

Stefano, G./Peteraf, M./Gianmiaro, V. (2010): Dynamic capabilities deconstructed: A bibliographic investigation into the origins, development, and future direction of the research domain, in: Industrial and Corporate Change, Jg. 19, Nr. 4, S. 1187-1204.

Thomas, DA. (2004): Diversity as strategy. In: Harvard Business Review, Jg. 82, 9, S. 98-98.

Tukker, A. (2004): Eight types of product service system; eight ways to sustainability? Experiences from SUSPRONET, in: Business Strategy and the Environment, Jg. 13, Nr. 4, S. 246-260.

Tukker, A./Tischner, U. (2006): Product-services as a research field: past, present and future, reflections from a decade of research, in: Journal of Cleaner Production, Jg.14, Nr. 17, S. 1552-1556. 
Tuli, K.R./Kohli, A.K./Bharadwaj, S.G. (2007): Rethinking customer solutions: from pro-duct bundles to relational processes, in: Journal of Marketing, Jg. 71, Nr. 3, S. 1-17.

Ulaga, W./Reinartz, W. (2012): Hybrid offerings: how manufacturing firms combine goods and services successfully, in: Journal of Marketing, Jg. 75, Nr. 6, S. 5-23.

Van Birgelen, M./de Ruyter, K./de Jong, A./Wetzels, M. (2002): Customer evaluations of after-sales service contact modes: an empirical analysis of national culture's consequences, in: International Journal of Research in Marketing, Jg. 19, Nr. 1, S. 43-64.

Matthyssens, P./ Vandenbempt, K. (1998): Creating competitive advantage in industrial services, in: Journal of Business \& Industrial Marketing, Jg. 13 Nr. 4/5, S. 339-355.

Vandermerwe, S./Rada, J. (1988): Servitization of business: adding value by adding services, in: European Management Journal, Jg. 6, Nr. 4, S. 314-324.

Voss, C.A. (2005): Paradigms of manufacturing strategy re-visited. International Journal of Operations \& Production Management, Jg. 25, Nr. 12, S. 1223-1227.

Windahl, C./Lakemond, N. (2006): Developing integrated solutions: the importance of relationships within the network, in: Industrial Marketing Management, Jg. 35, Nr. 7, S. 806-818.

Wise, R./Morrison, D. (1999): Beyond the exchange-the future of B2B, in: Harvard Business Review, Jg. 78, Nr. 6, S. 86-96.

Wise, R./Baumgartner, P. (1999): Go downstream: the new imperative in manufacturing. Harvard Business Review, Jg. 77, Nr. 5, S. 133-141.

Heiko Gebauer, PD Dr., ist Gruppenleiter an der Eidgenössischen Anstalt für Wasser, Abwasser und Gewässer (eawag), Lehrbeauftragter der Universität St.Gallen und Professor am Center for Service Research der schwedischen Universität Karlstad.

Anschrift: Eawag, Überlandstrasse 133, CH-8600 Dübendorf, Tel.: +41 (0)58/765-5484, Fax:+41 (0)58/765-5802, E-Mail: heiko.gebauer@eawag.ch

Caroline Saul, ist wissenschaftliche Mitarbeiterin an der Eidgenössischen Anstalt für Wasser, Abwasser und Gewässer (eawag).

Anschrift: Eawag, Überlandstrasse 133, CH-8600 Dübendorf, Tel.: +41 (0)58/765-5441, Fax: +41 (0)58/765-5802, E-Mail: caroline.saul@eawag.ch 